\title{
Mucocele mimetizando neoplasia glândular em região do palato: relato de caso
}

Mucocele mimetizing glandular neoplasm in the palate region: case report

Mucocele mimetizando neoplasma glandular en la región del paladar: reporte de caso

Rywendher dos Santos de OLIVEIRA ${ }^{\mathbf{1}}$

Tiago Novaes PINHEIRO ${ }^{2}$

Lioney Nobre CABRAL ${ }^{3}$

${ }^{1}$ Acadêmico do Curso de Graduação em Odontologia, Escola Superior de Ciências da Saúde, Universidade do Estado do Amazonas (UEA) 69065-001 Manaus-AM, Brasil

${ }^{2}$ Mestre e Doutor em Patologia Bucal pela Faculdade de Odontologia de Bauru, Universidade de São Paulo (USP), Professor da Escola Superior de Ciências da Saúde,Universidade do Estado do Amazonas (UEA) 69065-001 Manaus-AM, Brasil ${ }^{3}$ Doutor em Biotecnologia pela Universidade Federal do Amazonas (UFAM),

Professor da Escola Superior de Ciências da Saúde, Universidade do Estado do Amazonas (UEA) 69065-001 Manaus-AM, Brasil

\section{Resumo}

A mucocele é considerada uma lesão comum da mucosa oral, que resulta da ruptura de um ducto de glândula salivar e extravasamento de mucina para dentro dos tecidos moles adjacentes. Frequentemente encontrada no lábio inferior, o presente trabalho descreverá um caso de mucocele em mucosa do palato, considerada um região atípica, para muitos autores. Paciente do gênero feminina, 51 anos de idade, melanoderma, foi encaminhada para a Policlínica odontológica da UEA, com hipótese diagnóstica de hemangioma. $\mathrm{Na}$ anamnese, paciente relatou tempo de evolução de 5 meses, ausência de sintomatologia dolorosa, negou possuir hábitos de sucção e não ter traumatizado a área durante a alimentação ou escovação, além de não possuir hábito tabagista, etilista ou fazer uso de prótese, e sem histórico de doença sistêmica. Diante das informações clinicas, estabelecemos hipóteses diagnósticas: Carcinoma Mucoepidermóide e Mucocele. O diagnóstico foi confirmado através de biopsia excisional da lesão e exame histopatológico. Portanto, mesmo se tratando de uma lesão simples, podemos nos deparar com dificuldade diagnóstica, quando a localização da lesão é incomum. Entretanto, cabe ressaltar a importância de uma análise diferencial do caso, que para o presente trabalho, foi de grande importância para se chegar ao diagnóstico e assim proporciona um tratamento eficaz para a paciente.

Descritores: Mucocele; Palato; Glândulas Salivares.

\section{Abstract}

Mucocele is considered a common lesion of the oral mucosa that results from the rupture of a salivary gland duct and mucin extravasation into adjacent soft tissues. Often found on the lower lip, this paper will describe a case of mucocele on the mucuous palate, which is considered to be an atypical region by many authors. The female patient, aged 51 , melanoderma, was referred to the Dental Polyclinic of UEA with a diagnostic hypothesis of hemangioma. In the anamnesis, the patient reported an evolution time of 5 months, absence of painful symptoms, denied having suction habits and traumatizing the area during feeding or brushing, in addition to not having a habit of smoking, excessive alcohol consumption or using a prosthesis and without a history of systemic disease. Given the clinical information, we established diagnostic hypotheses: Mucoepidermoid Carcinoma and Mucocele. The diagnosis was confirmed by a biopsy of the lesion and histopathological examination. Therefore, even in the case of a simple lesion, we may find difficulty in diagnosing when the location of the lesion is unusual. However, it is worthy highlighting the importance of a differential analysis of the case, which for the present paper, was of great importance for coming up to the diagnosis, and thus providing an effective treatment for the patient.

Descriptors: Mucocele; Palate; Salivary Glands.

\section{Resumen}

El mucocele se considera una lesión común de la mucosa oral, que resulta de la ruptura de un conducto de la glándula salival y fuga de mucina en los tejidos blandos adyacentes. A menudo se encuentra en el labio inferior. El presente trabajo describirá un caso de mucocele en la mucosa del paladar, considerado una región atípica, para muchos autores. Paciente de 51 años, melanoderma, fue derivada a la clínica dental UEA, con una hipótesis diagnóstica de hemangioma. En la anamnesis, el paciente informó un tiempo de evolución de 5 meses, ausencia de síntomas dolorosos, negado tener hábitos de succión y no haber traumatizado el área durante la alimentación o el cepillado, No tengo el hábito de fumar, no bebo alcohol ni uso una prótesis y no tengo antecedentes de enfermedad sistémica. Given the clinical information, we established diagnostic hypotheses: Mucoepidermoid Carcinoma and Mucocele. El diagnóstico se confirmó mediante biopsia por escisión de la lesión y examen histopatológico. Por lo tanto, incluso en el caso de una lesión simple, podemos encontrar dificultades en el diagnóstico, cuando la ubicación de la lesión es inusual. Sin embargo, vale la pena enfatizar la importancia de un análisis diferencial del caso, que para el presente estudio fue de gran importancia para alcanzar el diagnóstico y, por lo tanto, proporciona un tratamiento efectivo para el paciente.

Descriptores: Mucocele; Paladar; Glándulas Salivales.

INTRODUÇÃO

As lesões císticas de glândulas salivares foram coletivamente designadas como mucoceles ${ }^{1}$, considerada uma lesão comum da mucosa oral, resultado da ruptura de um ducto de glândula salivar e o extravasamento de mucina para dentro dos tecidos moles adjacentes. Diferente de alguns cistos do ducto salivar, a mucocele não é um cisto verdadeiro sendo considerada um pseudocisto, pelo fato de não ser revestida por uma parede epitelial ${ }^{2}$.

A mucocele é desenvolvida por um mecanismo de extravasamento de muco, que geralmente é de origem traumática ou um fenômeno de retenção de muco causado por obstrução do ducto de uma glândula salivar. Esse extravasamento é causado pelo vazamento de líquido dos ductos ou ácinos de glândulas salivares nos tecidos circundantes. $O$ fenômeno de retenção ocorre devido uma abertura ductal reduzida, geralmente por causa de uma inflamação ou sialólito, causando a dilatação ductal e inchaço da superfície ${ }^{3}$.

A mucocele por extravasamento de muco tem maior frequência no lábio inferior e é menos frequente na mucosa jugal, assoalho bucal, trígono retromolar, lábio superior e palato. 
Afeta com maior frequência pacientes jovens, porém não há predileção por sexo, entretanto, o gênero feminino é o mais afetado ${ }^{4-6}$. O fenômeno de extravasamento representa mais de $80 \%$ de todas as mucoceles, são de fato pseudocistos que não possuem uma parede bem definida e são compostos de elementos comprimidos da superfície do tecido conjuntivo e componentes inflamatórios ${ }^{3,7,8}$. Por outro lado, a mucocele de retenção de muco é mais comum em pessoas de 40 anos de idade, sendo mais frequente no sexo feminino. Pode ocorrer em toda a mucosa oral, porém é mais comum na mucosa jugal ${ }^{9-11}$. As mucoceles de retenção consistem em uma cavidade cística bem definida com presença de parede epitelial forrada com células escamosas $3,7,8$.

As lesões superficiais da mucocele costumam se apresentar como inchaço elevado dos tecidos moles, com uma coloração azulada, enquanto as lesões mais profundas são mais nodulares, não apresentam vesícula e têm cor de mucosa normal ${ }^{12}$. A cor azul é causada por vaso-congestão celular e cianose tecidual do tecido acometido, observando-se acúmulo de fluido abaixo do tecido epitelial ${ }^{3,13,14}$.

Em relação a localização, as mucoceles ocorrem com maior freqüência no lábio inferior, por ser região susceptível a traumatismos diversos. Em um estudo feito por Oliveira et al. ${ }^{15}$, a partir de 112 casos de mucoceles orais diagnosticadas no Departamento de Patologia Oral da Faculdade de Odontologia de Bauru, 67 foram observadas em lábio inferior, compondo mais de $50 \%$ da amostra. Cohen ${ }^{16}$ relatou que $82 \%$ das mucoceles foram encontradas no lábio inferior, $8 \%$ mucosa bucal, $3 \%$ na área retromolar e $1 \%$ no palato.

Quanto a forma de tratamento existe várias formas, sendo que a escolha é baseada conforme a idade do paciente, considerando também a localização, tamanho e profundidade da lesão e o trauma que levou àquela condição. A cirurgia de exérese total é a mais comum e usual ${ }^{17}$. Sendo que alguns casos de mucoceles são auto limitantes, com a lesão se rompendo e cicatrizando sozinha ${ }^{2}$.

Nesse contexto, o presente trabalho tem por finalidade relatar um caso de mucocele localizada em palato duro, considerada por muitos autores uma localização atípica da lesão, e assim possibilitar um conhecimento mais amplo quanto a esta localização incomum. Também dispor as características clínicas da condição, onde mimetizava uma neoplasia de glândula salivar. Por fim, será abordado a decisão clínica tomada para se chegar ao diagnóstico e conduta terapêutica.
CASO CLÍNICO

Paciente do sexo feminino, 51 anos de idade, melanoderma, encaminhada à Policlínica Odontológica da UEA, com queixa principal, "uma bolinha no céu da boca", e hipótese diagnóstica de hemangioma. $\mathrm{Na}$ anamnese, relatou tempo de evolução da lesão de 5 meses, sem sintomatologia dolorosa, mencionou ainda não possuir hábitos de sucção e não ter traumatizado o local durante a alimentação ou escovação, além de não ser tabagista, etilista ou fazer uso de próteses removíveis, não sendo acometida de nenhuma doença sistêmica.

Ao exame clínico foi observado uma lesão em cúpula na região do palato duro esquerdo próximo a tuberosidade da maxila com coloração semelhante a fibromucosa circunjacente, tendo em seu centro uma mudança de coloração, padrão violáceo de natureza potencialmente vascular (Figura 1), que levou à hipótese diagnostica de uma lesão vascular. Solicitou-se uma tomografia, que revelou não alteração do tecido ósseo palatino relacionado à lesão (Figura 2).

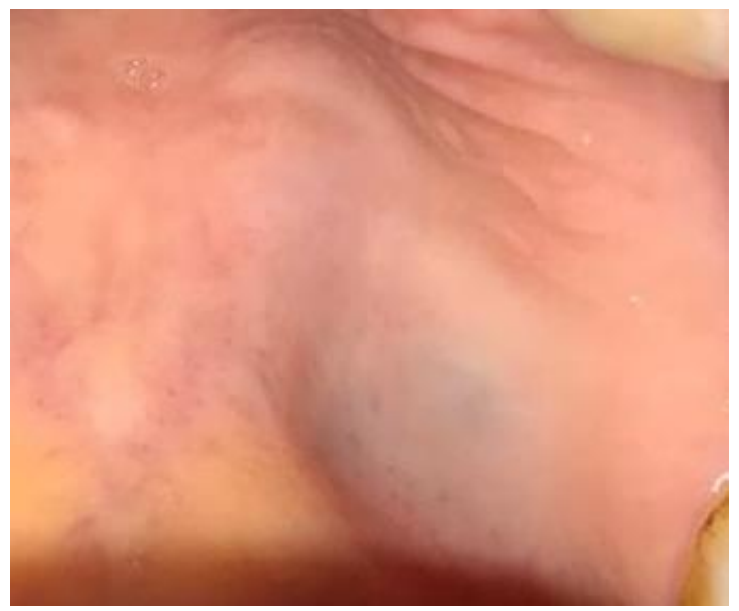

Figura 1: Imagem inicial da lesão.

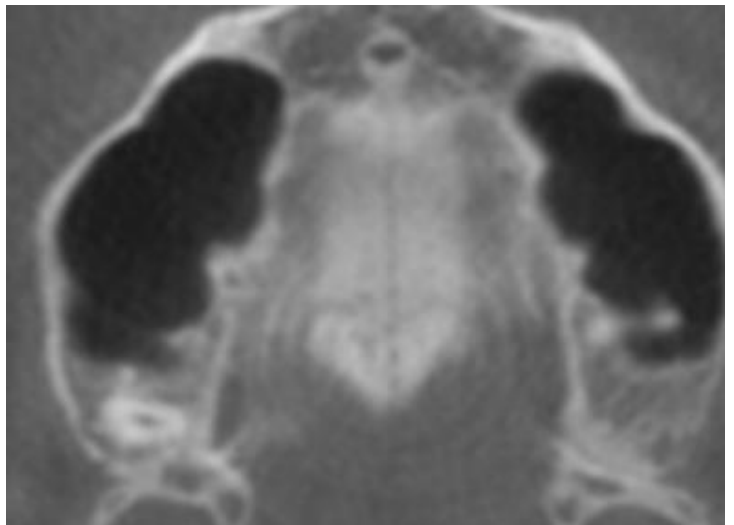

Figura 2: Tomografia computadorizada da maxila.

Para primeira abordagem clínica foi planejado a realização de uma punção aspirativa da lesão. $\mathrm{Na}$ execução do procedimento, realizou-se a técnica anestésica de bloqueio do nervo palatino maior esquerdo e complemento com infiltrativa à distância da 
lesão, e então feito a punção aspirativa, onde foi coletado um líquido incolor mucóide, com presença de sangue (Figura 3). A partir desta punção foi descartada a hipótese de lesão vascular, devido a presença de saliva na lesão, partindo para hipótese de cisto de extravasamento ou cisto de retenção mucoso (Mucocele) e neoplasia glandular (Carcinoma mucoepidermóide). Foi realizado o esfregaço do material em duas laminas de vidro, fixado com solução de Álcool-Éter e armazenado em um frasco. (Figura 4), para ser encaminhado ao laboratório de Patologia da UEA para análise citopatológica. No exame citopatológico 0 esfregaço revelou um material eosinófilico amorfo de aspecto mucinoso, de coloração PAS positiva, coloração esta capaz de corar a mucina presente na saliva. Nota-se também eventuais células inflamatórias mononucleadas e hemácias distribuídas aleatoriamente (Figura 5). Resultando um diagnóstico sugestivo de cisto de extravasamento mucoso (mucocele).

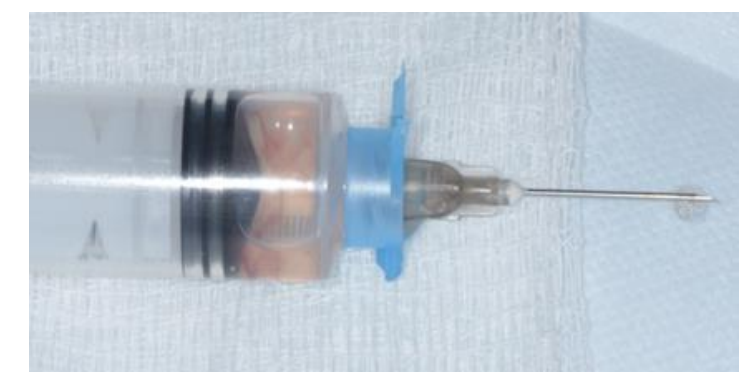

Figura 3: Imagem evidenciando o líquido coletado na punção.

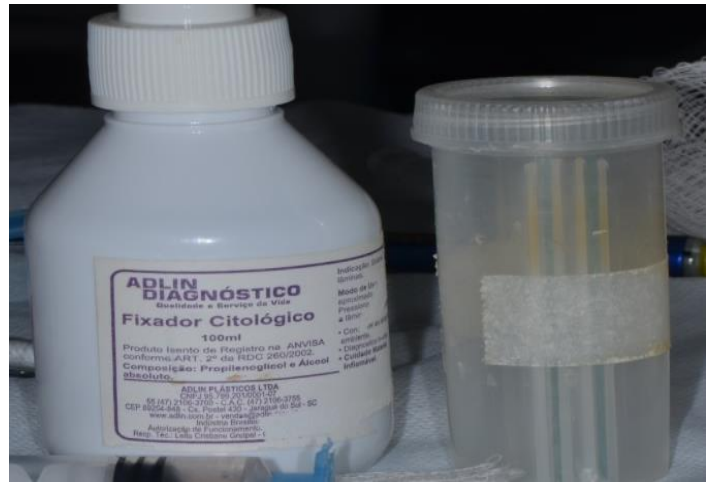

Figura 4: Imagem do material usado para realização do esfregaço citológico.

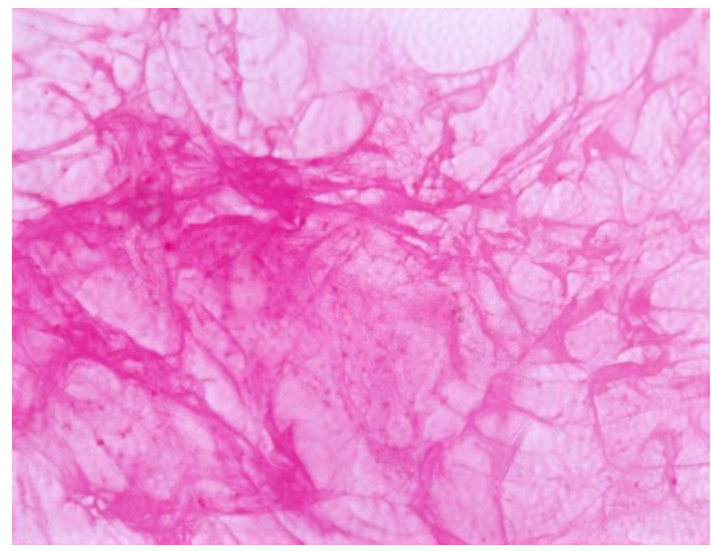

Figura 5: Imagem microscópica da análise citopatológica.
Para concluir o diagnóstico, planejamos uma biopsia incisional da lesão. O motivo de escolha do tipo de biopsia foi o fato do diagnóstico ser sugestivo de um carcinoma mucoepidermoide, visto o exame citopatológico não ser conclusivo, porém muito útil para condução cirúrgica, e este planejamento alterado para excisional no transoperatório pela suposta benignidade durante 0 ato. Foram então solicitados da paciente, exames laboratoriais (hemograma completo, glicemia em jejum, coagulograma e ureia).

Após avaliação dos exames solicitados, e estes dentro do padrão de normalidade. Realizamos a biopsia, com técnica anestésica de bloqueio do nervo palatino maior esquerdo, complementada com infiltrativa à distancia da lesão para não edemaciar o tecido. A incisão foi executada com um bisturi 15 no formato de um "V" invertido, depois foi realizada a divulsão tecidual com remoção de toda a lesão, bem como das glândulas salivares menores associadas (Figura 6). Logo após, foi realizada a sutura, com fio 0.4 de nylon, ponto simples.

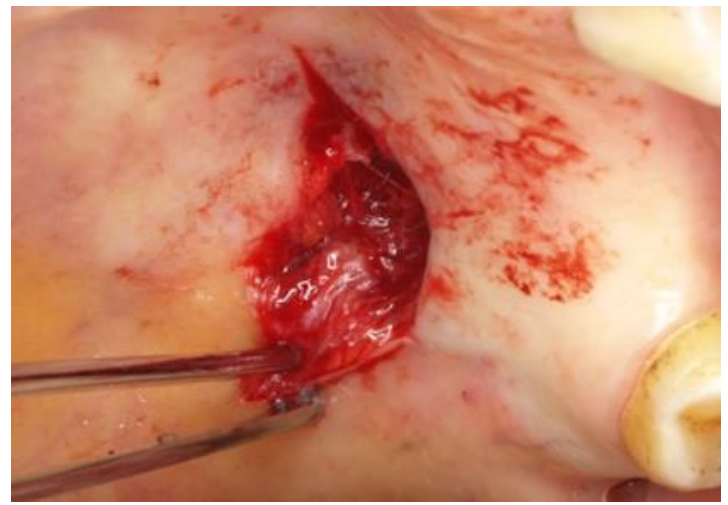

Figura 6: Fotografia do campo cirúrgico após remoção da lesão

A amostra do tecido removido media cerca de $8 \mathrm{~mm}$ (Figura 7), esse material sendo armazenado em um recipiente com formol a $10 \%$ e encaminhado ao laboratório de patologia da UEA para análise.

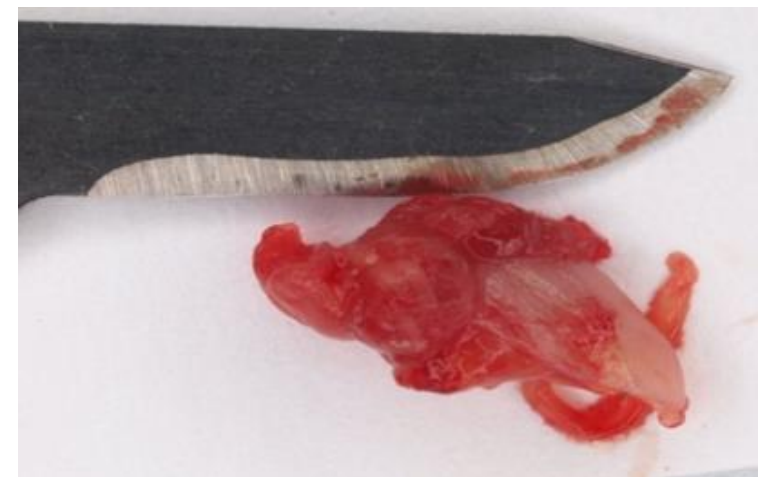

Figura 7: Fotografia evidenciando o tecido coletado na biopsia incisional.

No exame histopatológico, cortes microscópicos revelaram fragmentos da mucosa bucal revestida por epitélio estratificado 
pavimentoso paraqueratinizado, com tecido conjuntivo subjacente frouxo não modelado, apresentando glândulas salivares menores com ductos dilatados e alguns rompidos, além de áreas focais de moderado infiltrado inflamatório mononuclear linfocitário periductal. Áreas de mucinose com macrófagos de permeio e exsudato fibrinoso (Figura $8 \mathrm{~A}$ e B). Concluindo o diagnóstico de cisto de extravasamento mucinoso (mucocele).
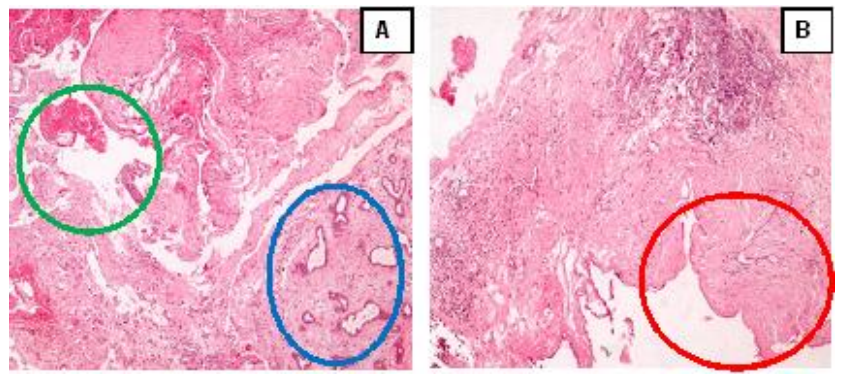

Figura 8: Imagem da microscopia Histopatológica. A, área de extravasamento de mucina circundado por tecido de granulação (verde), bem como ductos de glândulas salivares menores dilatados, alguns obstruídos (azul). B, imagem evidenciando todo corte histológico onde se consegue observa 0 infiltrado inflamatório mononuclear (vermelho).

Sete dias após a cirurgia a paciente retornou, sendo realizada a remoção da sutura e observada uma boa cicatrização da lesão, mostrando um bom prognóstico (Figura 9). Após 2 meses, a paciente retornou para nova proservação, sendo observada nenhuma recidiva e um bom reparo do tecido fibromucoso palatino (Figura 10).

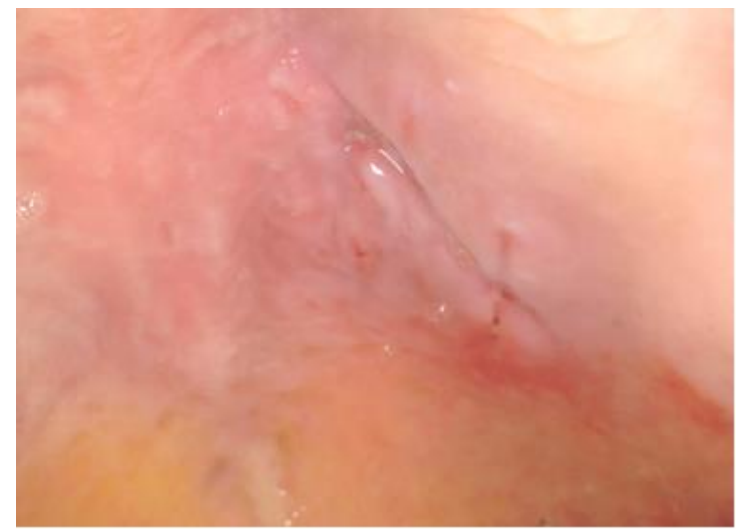

Figura 9: Pós-operatório, campo cirúrgico após 7 dias.

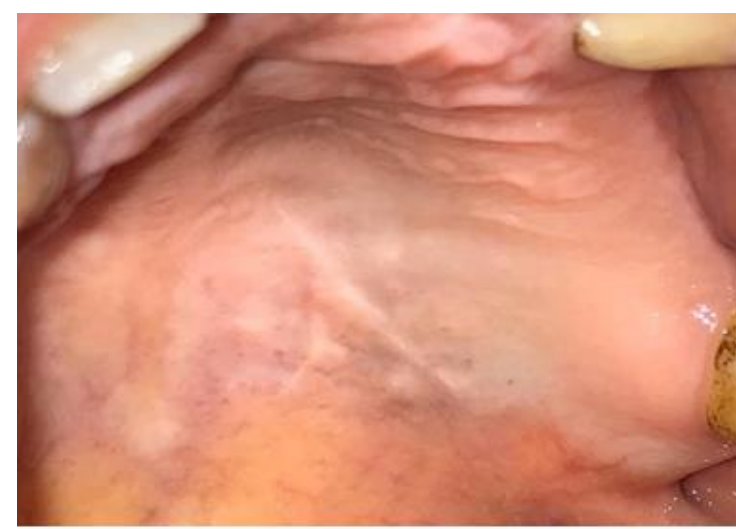

Figura 9: Pós-operatório, campo cirúrgico após 2 meses.
Após 7 meses, paciente retornou para novo atendimento de proservação, onde pode ser observado uma boa evolução e não recidiva da lesão. Portanto, paciente recebeu alta, com tratamento concluído com êxito.

DISCUSSÃO

A mucocele é uma lesão considerada comum da mucosa oral, que pode afetar a população como um todo. Muitas das vezes surge de uma alteração das glândulas salivares menores que se dá por um acúmulo de líquido na mucosa ${ }^{4}$. Yamasoba et al. $^{17}$ destacaram dois fatores etiológicos na mucocele: traumatismo e obstrução dos ductos da glândula salivar. A mucocele por extravasamento é causada por um vazamento de fluídos dos ductos ou ácinos para os tecidos circunjacentes. Tal fenômeno é comumente encontrado nas glândulas salivares menores ${ }^{4}$.

O diagnóstico quando de extravasamento quase sempre é baseado em achados clínicos, com aspecto patognomônico ${ }^{18}$, sendo à localização da lesão, história de trauma, aparência rápida, variações de tamanho, cor azulada e consistência, alguns dos fatores importantes a serem considerados antes do diagnóstico final. A citologia aspirativa por agulha fina demonstra o fenômeno de retenção de muco. A análise química pode revelar presença de proteínas e alto teor de amilase. A localização e determinação da origem da lesão podem ser feitas em algumas condições por tomografia computadorizada ${ }^{17}$. Nosso caso foi utilizado todos esses meios citados na literatura, que foram importantes para conclusão diagnóstica, mesmo diante da localização e aspecto incomum da lesão. De acordo com um estudo feito por Oliveira et al. ${ }^{15}$ a partir de 112 casos de mucoceles orais diagnosticadas no Departamento de Patologia Oral da Faculdade de Odontologia de Bauru, 67 foram observadas em lábio inferior, compondo mais de $50 \%$ da amostra. Cohen ${ }^{16}$ relatou que $82 \%$ das mucoceles foram encontradas no lábio inferior, $8 \%$ mucosa bucal, $3 \%$ na área retromolar e somente $1 \%$ no palato.

O tratamento convencional das mucoceles orais é a extirpação cirúrgica do cisto da mucosa circundante e do tecido glândular. Com uma simples incisão, o conteúdo seria drenado, mas a lesão reapareceria à medida que a ferida cicatrizasse ${ }^{19}$. No nosso caso, não houver recidiva da lesão após a realização da biópsia excisional da lesão, por havemos retirado o tecido glandular comprometido, para fins de definição diagnóstica. Destarte, pode-se considerar que em certos casos de mucoceles a 
biópsia pode servir como tratamento eficaz, como descrito em nosso caso.

A excisão cirúrgica com remoção das glândulas salivares menores envolvidas tem sido sugerida como tratamento. $O$ tecido excisado deve ser submetido às investigações patológicas para confirmar o diagnóstico e descartar o tumor da glândula salivar. Em nosso caso o tecido foi encaminhado para análise no Serviço de Patologia Bucal da UEA. A ablação a laser, criocirurgia e eletrocautério são abordagens que também podem ser utilizadas para o tratamento da mucocele convencional com sucesso variável ${ }^{17}$.

\section{CONCLUSÃO}

Conclui-se que mesmo se tratando de uma condição simples, podemos nos deparar com a dificuldade diagnóstica, quando sua localização é incomum. Daí a importância da análise para orientação da abordagem cirúrgica com o fim da avaliação histopatológica, estes passos, sendo fundamentais na estruturação propedêutica, conclusão diagnóstica e terapêutica da condição, a paciente não apresentando recidiva da lesão.

\section{REFERÊNCIAS}

1. Tal $\mathrm{H}$, Altini $M$, Lemmer J. Multiple mucous retention cysts of the oral mucosa. Oral Surg Oral Med Oral Pathol. 1984;58(6):692-95.

2. Baurmash HD, Caltado E, Mosadoni A, et al. Patologia das glândulas salivares: mucocele. In: Neville BW, Damm DD, Allen C, CHI A. 4a ed. Rio de Janeiro: Elsevier; 2016, p. 456-458.

3. Baurmash HD. Mucoceles and ranulas. J Oral Maxillofac Surg. 2003;61(3):369-78.

4. Bagán Sebastián JV, Silvestre Donat FJ, Peñarrocha Diago $M$, Milián Masanet MA. Estudio clínico-patológico de los mucoceles de la cavidad oral [Clinico-pathological study of oral mucoceles]. Av Odontoestomatol. 1990;6(7):389-91, 394-95.

5. Kang SK, Kim KS. [Clinical and histopathologic study of salivary mucoceles]. Taehan Chikkwa Uisa Hyophoe Chi. 1989;27(11):1059-71.

6. Mc Donald JS. Tumores dos tecidos moles da boca e cistos e tumores dos ossos. In: Mc Donald RE, Avery DR. Odontopediatria, 6.ed. Rio de Janeiro: Guanabara Koogan; 1995. p.111-28.

7. Harrison JD. Salivary mucoceles. Oral Surg Oral Med Oral Pathol. 1975;39(2):268-78.

8. Cataldo E, Mosadomi A. Mucoceles of the oral mucous membrane. Arch Otolaryngol. 1970;91(4):360-65.

9. Bodner L, Tal H. Salivary gland cysts of the oral cavity: clinical observation and surgical management. Compendium. 1991;12(3):150, 152,154-56.
10. Kakarantza-Angelopoulou E, Triantaphyllou A. Blennodeis kysteis apo katakratese. (Mia spania poikilia blennokeles) [Mucous retention cysts of the minor salivary glands. A specific type of mucocele]. Odontostomatol Proodos. 1989;43(4):373-9.

11. Seifert G, Donath K, von Gumberz C. [Mucoceles of the minor salivary glands. Extravasation mucoceles (mucus granulomas) and retention mucoceles (mucus retention cysts). HNO. 1981;29(6):179-91.

12. Koudelka BM. Obstructive disorders. In: Ellis GL, Auclair PL, Gnepp DR, editors. surgical pathology of the salivary glands. Philadelphia WB: Saunders Company; 1991, p. 26-39.

13. Bentley JM, Barankin B, Guenther LC. A review of common pediatric lip lesions: herpes simplex/recurrent herpes labialis, impetigo, mucoceles, and hemangiomas. Clin Pediatr (Phila). 2003;42(6):475-82.

14. Tran TA, Parlette HL 3rd. Surgical pearl: removal of a large labial mucocele. J Am Acad Dermatol. 1999;40(5 Pt 1):760-2.

15. Oliveira DT, Consolaro A, Freitas FJ. Histopathological spectrum of 112 cases of mucocele. Braz Dent J. 1993; 4(1):29-36.

16. Cohen L. Mucoceles of the oral cavity. Oral Surg Oral Med Oral Pathol. 1965;19:365-72.

17. Yamasoba T, Tayama N, Syoji M, Fukuta $M$. Clinicostatistical study of lower lip mucoceles. Head Neck. 1990;12(4):316-20.

18. Yagüe-García J, España-Tost AJ, Berini-Aytés L, Gay-Escoda C. Treatment of oral mucocelescalpel versus $\mathrm{CO} 2$ laser. Med Oral Patol Oral Cir Bucal. 2009;14(9):e469-74.

19. Huang IY, Chen CM, Kao YH, Worthington P. Treatment of mucocele of the lower lip with carbon dioxide laser. J Oral Maxillofac Surg. 2007;65(5):855-58.

\section{CONFLITO DE INTERESSES}

Os autores declaram não haver conflitos de interesse

\section{AUTOR PARA CORRESPONDÊNCIA}

\section{Lioney Nobre Cabral}

Professor da Escola Superior de Ciências da Saúde, Universidade do Estado do Amazonas (UEA)

69065-001 Manaus-AM, Brasil

E-mail: lioneycabral@yahoo.com.br 